\title{
Étude expérimentale de la contamination d'un sédiment et d'une annélide polychète (Nereis diversicolor Muller) par le zinc 65
}

\author{
J. P. BAUDIN $(*)$ \\ (Manuscrit reçu le 10 décembre 1980)
}

\begin{abstract}
RÉSUMÉ
Radionucléide à vie moyenne ( $\mathrm{T} \simeq 245$ jours), le zinc 65 , qui est un isotope d'un oligoélément biologique, est présent dans certainș effluents liquides rejetés par les installations nucléaires. La première partie de cette étude est consacrée au transfert du zinc 65 entre l'eau et le sédiment. Ce processus se caractérise essentiellement par sa rapidité et son intensité. En 48 heures l'eau perd $50 \mathrm{p}$. cent de son activité et le transfert approche $100 \mathrm{p}$. cent au-delà du $20^{\circ}$ jour. Le zinc 65 est fortement lié au sédiment et sa désorption est quasiment inexistante.

Dans la deuxième partie l'auteur expose les résultats de la contamination par le zinc 65 de Nereis diversicolor, espèce qui peut être considérée comme un vecteur de " remise en circulation " du radionucléide fixé dans le sédiment. Cette hypothèse se trouve en partie vérifiée, mais il apparaît que l'accumulation du zinc 65 par Nereis diversicolor est 20 fois plus importante à partir de l'eau qu'à partir du sédiment.
\end{abstract}

\begin{abstract}
Zinc 65 is a radionuclide with an intermediate half-life ( 245 days). It is an isotope of a biological trace element which can be found in some liquid wastes released by nuclear facilities. The first part of the paper concerns zinc 65 transfer from water to sediment. This process is essentially characterised by its rapidity and intensity. After 48 hours water loses $50 \mathrm{p}$. cent of its activity and transfer reaches 100 p. cent beyond the 20 th day. Zinc 65 is strongly bound to the sediment and its desorption is almost non-existent.

The second part reports the results about zinc 65 contamination of Nereis diversicolor, a species which can be considered as a vehicle of recirculation for the radionuclide fixed in the sediment. This assumption is partly verified although zinc 65 accumulation by Nereis diversicolor from water is 20 times more important than from sediment.
\end{abstract}

${ }^{*}{ }^{*}$ Chargé de Recherche au C.N.R.S., Commissariat à l'Énergie atomique, Institut de Protection et de Sûreté nucléaire, Département de Protection, S.E.R.E./L.E.P.E., B. P. n ${ }^{\circ} 1,13115$ Saint-Paul-lez-Durance. 


\section{INTRODUCTION}

- Parmi tous les rądionucléides rejetés dans le milieu naturel du fait de l'utilisation de l'énergie nucléaire, le zinc 65 présente un intérêt particulier car c'est un isotope d'un élément qui a un rôle essentiel chez la plupart des espèces animales et végétales [1-5]. Ce radionucléide est l'un de ceux qui sont fréquemment décelés dans les eaux et les organismes aquatiques à la suite des explosions atomiques et dans les aires d'épandage des effluents liquides issus des installations nucléaires [6-11]. L'étude du transfert du zinc 65 entre les différents composants des écosystèmes aquatiques apparalt donc très importante au plan de la protection sanitaire.

Dans le cadre d'un travail expérimental portant sur un écosystème saumâtre, nous avons étudié les échanges du zinc 65 entre l'eau et le sédiment, puis la fixation du radionucléide par une espèce benthique, Nereis diversicolor, qui est une annélide polychète très euryhaline.

\section{1. ÉCHANGES DU ZINC 65 ENTRE L'EAU ET LE SÉDIMENT}

\subsection{Protocole expérimental}

L'eau et le sédiment utilisés (tableau I) proviennent de l'étang de Citis, petit étang saumâtre situé sur la bordure occidentale de l'étang de Berre [12]. Les expériences sont réalisées dans des séries de bocaux en verre dans lesquels sont

TABLEAU I

PRINCIPAUX CARACTĖRES CHIMIQUES

DE L'EAU ET DU SÉDIMENT UTILISÉ

\begin{tabular}{|c|c|c|c|}
\hline \multicolumn{2}{|c|}{$\underset{\left(\mathrm{mg} .1^{-1}\right)}{\mathrm{Eau}}$} & \multicolumn{2}{|c|}{$\begin{array}{c}\text { Sédiment } \\
\text { Éléments échangeables } \\
\text { (mg/100 g de sédiment } \mathrm{sec})\end{array}$} \\
\hline $\mathrm{HCO}_{3}^{-} \ldots \ldots \ldots \ldots$ & 214 & $\mathrm{Na}^{+} \ldots \ldots \ldots \ldots \ldots$ & 375 \\
\hline $\mathrm{Cl}^{-} \ldots \ldots \ldots \ldots$ & 8100 & $\mathrm{~K}^{+} \ldots \ldots \ldots \ldots$ & 182,5 \\
\hline $\mathrm{Ca}^{++} \ldots \ldots \ldots \ldots$ & 660 & $\mathrm{Ca}^{++} \ldots \ldots \ldots \ldots$ & 837.5 \\
\hline $\mathrm{Mg}^{++} \ldots \ldots \ldots \ldots$ & 945 & $\mathrm{Mg}^{++} \ldots \ldots \ldots \ldots$ & 275 \\
\hline $\mathrm{Na}^{+} \ldots \ldots \ldots \ldots \ldots$ & 3780 & $\mathrm{Mn}^{++} \ldots \ldots \ldots \ldots$ & 3 \\
\hline $\mathrm{K}^{+} \ldots \ldots \ldots \ldots \ldots$ & 220 & $\mathrm{Cl}^{-} \ldots \ldots \ldots \ldots$ & 1100 \\
\hline $\mathrm{NO}_{3}^{-} \ldots \ldots \ldots \ldots \ldots$ & 0,05 & $\mathrm{Zn}^{++} \ldots \ldots \ldots \ldots$ & 0,25 \\
\hline $\mathrm{NO}_{2}^{-} \ldots \ldots \ldots \ldots \ldots$ & 0,16 & $\mathrm{SiO}_{2} \ldots \ldots \ldots \ldots$ & 1,5 \\
\hline $\mathrm{NH}_{4}^{+} \ldots \ldots \ldots \ldots$ & 0,20 & $\mathrm{PO}_{4}^{-}-\ldots \ldots \ldots \ldots$ & 50 \\
\hline $\mathrm{PO}_{4}^{---} \ldots \ldots \ldots \ldots$ & 0,50 & $\mathrm{SO}_{4}^{--} \ldots \ldots \ldots \ldots \ldots$ & 457,5 \\
\hline $\begin{array}{l}\mathrm{SO}_{4}^{--} \cdots \cdots \cdots \cdots \cdots \\
\mathrm{Zn}^{++}\end{array}$ & 2300 & & \\
\hline & 0,010 & & \\
\hline
\end{tabular}

introduits $250 \mathrm{~g}$ de sédiment humide, formant une couche de $2 \mathrm{~cm}$ d'épaisseur, puis $11 \mathrm{~d}$ 'eau constituant une colonne de $10 \mathrm{~cm}$ de hauteur. La contamination du milieu est effectuée par l'apport de 1,2 à $1,3 \mu \mathrm{Ci}$ de zinc 65 en solution acide, 
ce qui représente environ 10 fois la C.M.A. population. Un agitateur en verre tournant à raison de 20 rotations par minute assure une bonne homogénéisation de l'eau sans que des particules ne se mettent en suspension.

L'adsorption du zinc 65 sur les parois des bocaux étant négligeable, la mesure d'échantillons d'eau prélevés régulièrement permet d'évaluer le passage du radionucléide de l'eau dans le sédiment. Dans l'étude de ce transfert, deux paramètres ont été pris en considération : la granulométrie du sédiment et la hauteur de la colonne d'eau surnageante. Le sédiment " fin ", récolté au centre de l'étang est composé de 2 p. cent de sable, 17,9 p. cent de limon grossier, 58,1 p. cent de limon fin, 8,8 p. cent d'argiles, et 13,4 p. cent de matières organiques. Le sédiment " grossier " qui provient des bords de l'étang est essentiellement constitué de sable et de petits cailloux - limons, argiles et matières organiques étant en très faible proportion.

\subsection{Résultats}

\subsubsection{Cinétique du transfert eau-sédiment}

La cinétique de la fixation par le sédiment du zinc 65 contenu dans l'eau présente, dans les trois cas étudiés, une très grande similitude (tableau II, fig. 1).

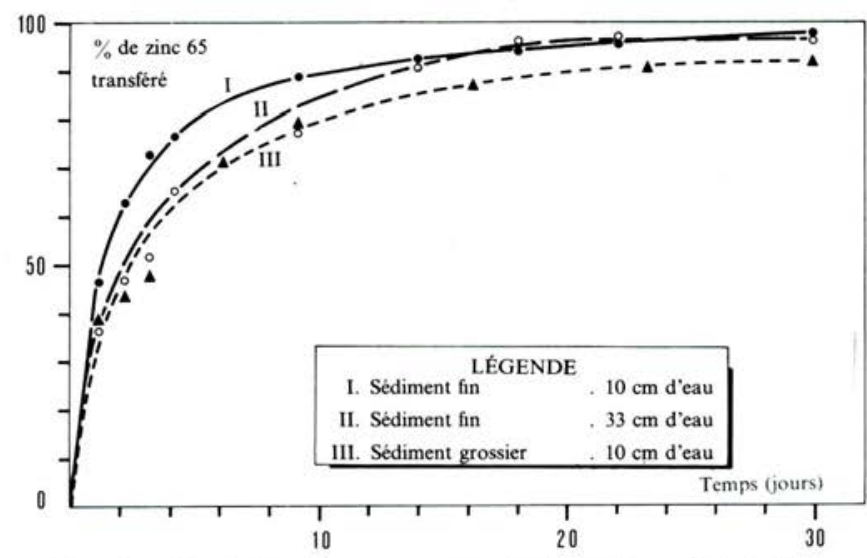

Fig. 1. - Évolution du pourcentage de zinc 65 transféré de l'eau au sédiment dans différentes conditions expérimentales.

Au cours des premiers jours, on assiste à un transfert extrêmement rapıde du radionucléide qui se traduit, dans le cas du sédiment fin par exemple (expérience A), par une perte d'environ 70 p. cent de l'activité initiale de l'eau.

Dans les deux autres cas, cette diminution est plus faible, de l'ordre de 50 p. cent. Par la suite, le phénomène se ralentit nettement pour devenir très lent quand la majeure partie du zinc 65 initialement contenu dans l'eau a été fixée dans le sédiment. 
Le déroulement du processus tel quie nous venons de le décrire montre que la vitesse du transfert du radionucléide décroît avec la diminution de l'activité de l'eau. Cette constatation est parfaitement illustrée dans les premiers jours de l'expérience. Dans le premier cas par exemple (sédiment fin, hauteur d'eau de $10 \mathrm{~cm}$ ), la proportion du zinc 65 présent dans l'eau qui passe dans le sédiment est de $46 \mathrm{p}$. cent le premier jour, $31 \mathrm{p}$. cent le deuxième, $25 \mathrm{p}$. cent le troisième et $15 \mathrm{p}$. cent le quatrième.

\section{TABLEAU II}

POURCENTAGE DE ZINC 65 FIXÉ DANS LE SÉDIMENT DANS LES DIFFÉRENTES CONDITIONS EXPÉRIMENTALES. A : Sédiment fin, hauteur d'eau de $10 \mathrm{~cm}$; B : Sédiment fin, hauteur d'eau de $33 \mathrm{~cm} ; \mathrm{C}$ : Sédiment grossier, hauteur d'eau de $10 \mathrm{~cm}$.

\begin{tabular}{|c|c|c|c|}
\hline $\begin{array}{c}\text { Temps } \\
\text { (jours) }\end{array}$ & A & B & C \\
\hline 1 & 46,2 & 36,4 & 39,2 \\
\hline 2 & 62,9 & 46,8 & 44,7 \\
\hline 3 & 72,3 & 51,9 & 47,8 \\
\hline 4 & 76,6 & 65,1 & - \\
\hline 6 & - & - & 72,6 \\
\hline 9 & 89 & 77,3 & 79,8 \\
\hline 14 & 93 & 91,6 & - \\
\hline 16 & - & - & 87,4 \\
\hline 18 & 94,9 & 96,1 & - \\
\hline 22 & 96 & 96,1 & - \\
\hline 23 & - & - & 90,2 \\
\hline 30 & 95,7 & 96,9 & 92,3 \\
\hline
\end{tabular}

La cinétique du transfert du zinc 65 est sensiblement la même dans les trois cas étudiés. On peut donc conclure que la granulométrie et la surface de contact entre l'eau et le sédiment n'ont pas une influence déterminante sur le processus. Cependant, des écarts apparaissent et on relève des différences maximales de l'ordre de 20 à $25 \mathrm{p}$. cent affectant le taux de zinc 65 transféré selon la granulométrie du sédiment, dans les premiers jours de l'expérience. Mais par la suite, ces écarts tendent à se réduire pour devenir très faibles $(4 \mathrm{p}$. cent après 30 jours. 


\subsubsection{Localisation du zinc 65 fixé dans le sédiment}

A l'issue de nos expériences, le sédiment apparaît donc comme un véritable piège pour le zinc 65 . Cette constatation est conforme aux teneurs en zinc stable mesurées dans l'étang de Citis, qui sont de l'ordre de $10 \mu \mathrm{g} / \mathrm{l}$ pour l'eau et de $11 \mathrm{mg} / \mathrm{kg}$ frais de sédiment. Elle correspond-également au fait que le processus de désorption du zinc 65 est quasiment inexistant. Ainsi, une étude réalisée selon le protocole décrit ( $c f .1 .1)$, mais au cours de laquelle le zinc 65 était préalablement introduit dans le sédiment, ne nous a pas permis de déceler le passage dans l'eau d'une quantité mesurable de radionucléide. On peut donc conclure que le zinc 65 a une très grande affinité pour le sédiment, conclusion qui rejoint les données tirées de divers travaux, notamment de ceux consacrés à la Columbia river, aux U.S.A. [13-15].

A la fin des expériences de transfert nous avons fait une étude sommaire de la répartition verticale du zinc 65 fixé dans le sédiment (série I : sédiment fin, hauteur d'eau de $10 \mathrm{~cm}$ ). Les teneurs en radiozinc mesurées sont de $97,1 \mathrm{nCi} / \mathrm{g}$ frais dans la couche superficielle (2 à $3 \mathrm{~mm}$ ), de $32,1 \mathrm{nCi} / \mathrm{g}$ frais dans la zone comprise entre -3 et $-5 \mathrm{~mm}$, de $5,2 \mathrm{nCi} / \mathrm{g}$ frais dans la zone comprise entre -5 et $-10 \mathrm{~mm}$ et de $0,1 \mathrm{nCi} / \mathrm{g}$ frais dans la zone comprise entre -10 et $-20 \mathrm{~mm}$. Malgré l'épaisseur différente des zones successives, la décroissance très nette de leur activité montre que le zinc 65 reste essentiellement localisé dans les couches supérieures du sédiment et que sa migration en profondeur est très lente.

\section{CONTAMINATION DE NEREIS DIVERSICOLOR}

La fixation du zinc 65 dans le sédiment apparaissant comme un processus quasiment irréversible, il nous a paru intéressant de savoir s'il y avait une possibilité biologique de " remise en circulation » d'une partie au moins du radionucléide fixé. A cet égard Nereis diversicolor nous a semblé représenter, dans l'écosystème étudié, un support éventuel à l'intégration dans les chaînes alimentaires du zinc 65 retenu dans le sédiment.

\subsection{Protocole expérimental}

Dans 10 béchers en verre contenant $250 \mathrm{~g}$ de sédiment $(3 \mathrm{~cm})$ et $100 \mathrm{ml}$ d'eau, nous avons introduit 5 à 6 vers dont le poids total est d'1 g environ. L'eau est contaminée par l'apport d'une solution de chlorure de zinc 65 représentant une activité de $1,4 \mu \mathrm{Ci}\left({ }^{1}\right)$. La cinétique de la contamination des annélides a été suivie par la mesure de l'activité de tous les individus d'une même bécher, à partir du $14^{\mathrm{e}}$ jour suivant l'introduction du radionucléide dans le milieu.

\subsection{RÉSUltats}

\subsection{1. Évolution de l'activité des différents composants}

Le zinc 65 introduit dans le milieu se fixe rapidement dans le sédiment et la décroissance de l'activité spécifique de l'eau ( fig. 2) est conforme aux résultats exposés précédemment.

( ${ }^{1}$ La concentration représente environ 100 fois la C.M.A. population.

vOL. $16-\mathrm{N}^{\circ} 3$ 
Ainsi, après 14 jours l'eau a perdu 93 p. cent de sa teneur en zinc 65 et le sédiment se trouve alors fortement contaminé. La représentation graphique de l'évolution de l'activité spécifique du sédiment ( fig. 2) est basée sur des valeurs théoriques, car nous avons opéré comme si la répartition du radionucléide était

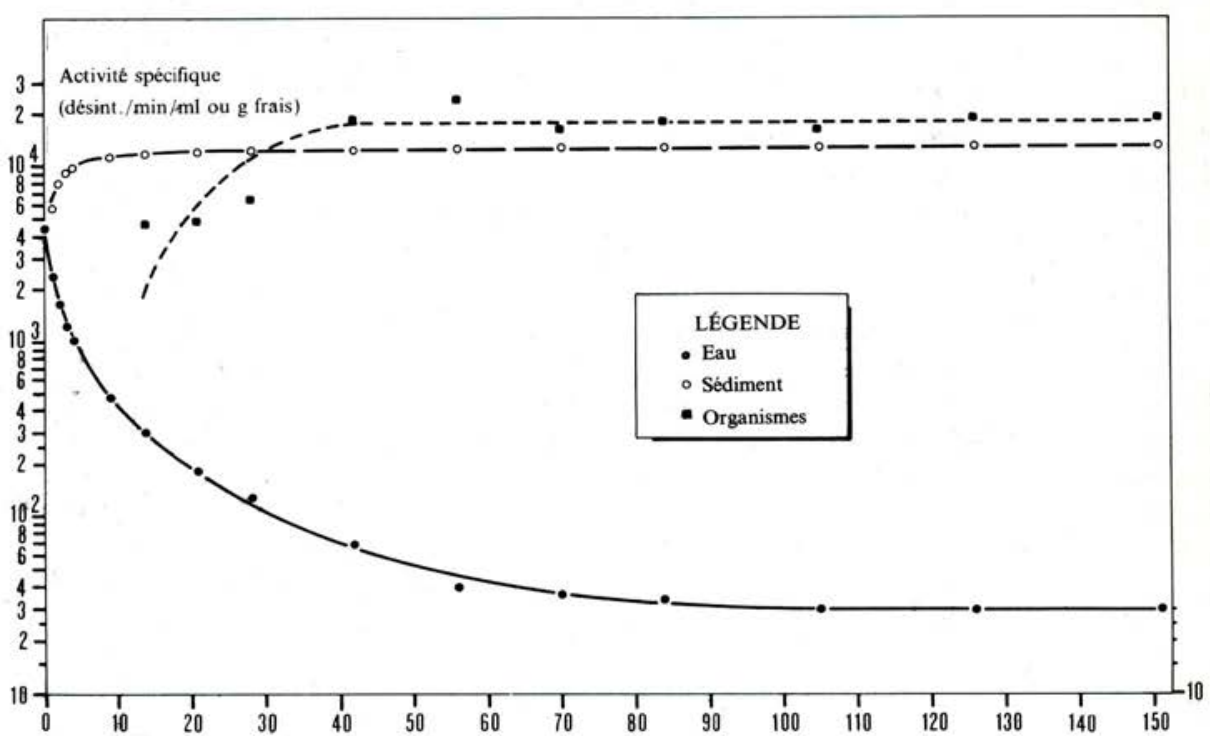

FIG. 2. - Évolution de l'activité spécifique de l'eau, du sédiment et des annélides.

homogène. Nous avons vu qu'en fait il en était autrement; mais dans cette expérience la présence des annélides permet cette approximation. En effet, l'activité de fouissage de Nereis diversicolor, même si elle est limitée, conduit à une relative homogénéisation de la répartition du zinc 65 dans le sédiment. Un tel phénomène a été observé par TRIQUET [16] et AMIARD-TRIQUET [17] au cours de l'étude de la contamination d'Arenicola marina par le cobalt 60, le cérium 144 et le fer 59 dont la fixation dans le sédiment est similaire à celle du radiozinc.

Lors du premier prélèvement, effectué au $14^{\mathrm{e}}$ jour de l'expérience, l'activité spécifique des annélides est de $2,15 \mathrm{nCi} / \mathrm{g}$ frais. Au cours des semaines suivantes, cette activité s'élève pour atteindre après 40 jours sa valeur maximale qui est de l'ordre de $8 \mathrm{nCi} / \mathrm{g}$ frais ( fig. 2). Par la suite, les fluctuations sont de faible amplitude, traduisant l'instauration d'un état d'équilibre entre la fixation et l'élimination du zinc 65 par les annélides. Au terme de l'expérience $(151 \mathrm{j})$ les vers ont fixé environ $0,5 \mathrm{p}$. cent du zinc 65 initialement introduit dans le milieu.

\subsubsection{Interprétation des résultats}

L'évolution de l'activité spécifique des annélides montre que la fixation du zinc 65 a lieu durant les 40 premiers jours de l'expérience, c'est-à-dire pendant la période au cours de laquelle le radionucléide se trouve en quantité notable dans 
TABLEAU III

ACTIVITÉ SPÉCIFIQUE DE L'EAU, DU SÉDIMENT ET DES ANNÉLIDES.

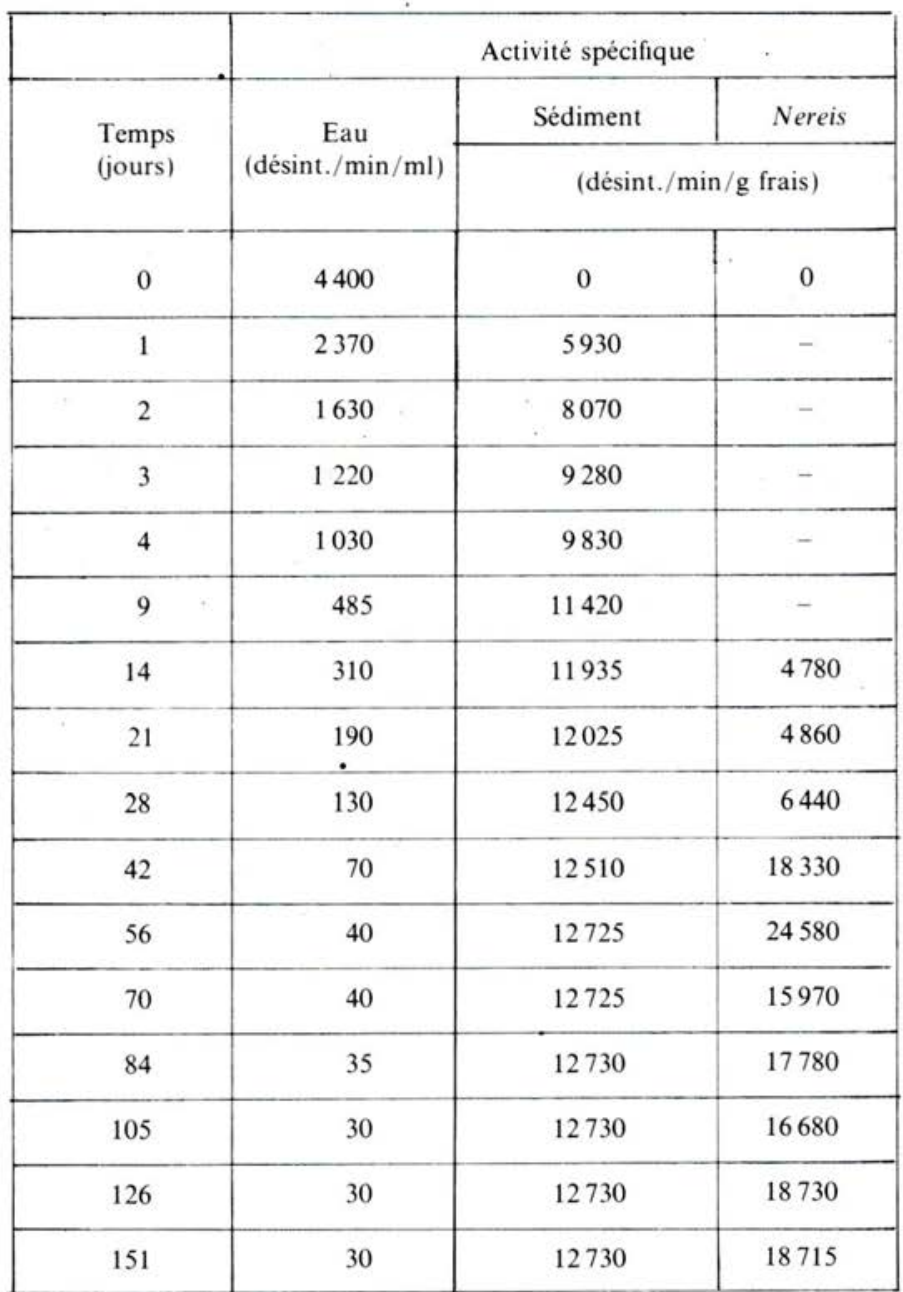

l'eau. Par la suite, l'activité spécifique reste remarquablement stable alors qu'une accumulation de zinc 65 à partir du sédiment, qui contient à ce moment-là $99 \mathrm{p}$. cent de la radioactivité initiale, devrait se traduire par une augmentation de ce paramètre. Ces faits conduisent donc à conclure que l'eau constitue le vecteur essentiel de la contamination de Nereis diversicolor par le zinc 65. Cette conclusion a été vérifiée en introduisant des annélides dans des récipients contenant $250 \mathrm{~g}$ de sédiment préalablement contaminé avec $2,1 \mu$ Cí de zinc 65 .

VOL. $16-\mathrm{N}^{\circ} 3$ 
Dans ces conditions, après 54 jours, l'activité spécifique des vers n'était que de $0,73 \mathrm{nCi} / \mathrm{g}$ frais, valeur environ 12 fois plus faible que celle obtenue au $40^{\mathrm{e}}$ jour de l'expérience précédente malgré une contamination plus élevée du milieu $(2,1 \mu \mathrm{Ci}$ contre $1,4 \mu \mathrm{Ci})$. Cette très nette prépondérance de l'eau a également été observée pour 4renicola marina, espèce pourtant limnivore, lors d'expériences de contamination avec le cobalt 60 , le cérium 144 et le fer 59 [16-17].

\section{CONCLUSION}

De nombreuses mesures in situ révèlent que le milieu sédimentaire en aval des installations nucléaires peut présenter de fortes teneurs en zinc $65[13,15,18$, 19]. Ces données sont en parfait accord avec la cinétique du transfert du radionucléide que nous avons mise en évidence et l'importance quantitative du processus. Sur la base de nos résultats et des travaux de différents auteurs, on peut penser qu'un taux élevé de sorption du zinc 65 sur un sédiment est observé quelles que soient sa granulométrie, ainsi que la température et la salinité de l'eau [20-22].

L'eau apparaît comme étant le vecteur le plus important de la contamination par le zinc 65 de Nereis diversicolor, bien que cette espèce soit en contact étroit avec le sédiment. Ce fait rejoint les conclusions tirées par CRoss et al. [23] et VILQUIN et al. [24] à l'issue de travaux concernant divers milieux sédimentaires. Ces auteurs ont démontré que les concentrations en zinc, manganèse et fer dans les annélides n'étaient pas liées à la concentration de ces éléments dans le sédiment. Cependant, ces constatations ne doivent pas masquer le fait que l'accumulation de zinc 65 par Nereis diversicolor à partir du sédiment est suffisante pour être à l'origine d'une contamination de la faune ichtyologique.

\section{BIBLIOGRAPHIE}

[1] Rice T. R. Review of zinc in Ecology. In : Radioecology (Schultz V. and A. W. Klement, Eds.), New York : Reinhold Publishing Corporation, 1963, 619-632.

[2] Valle B. L. Biochemistry, physiology and pathology of zinc. Physiol. Rev., 1959, 39, 443-490.

[3] Zirino A., Healy M. L. Inorganic zinc complexes in seawater. Limnol. Oceanogr., 1970, 15, 956-958.

[4] Wolfe D. A. Zinc enzymes in Crassostrea virginica. J. Fish. Res. Bd. Canada, 1970, 27, 59-69.

[5] UNDERWOOD E. J. Zinc. In : Trace elements in human and animal nutrition. New York : Academic press, 1971.

[6] Lowman F. G., Palumbo R., South D. J. The occurence and distribution of radioactive nonfission products in plants and animals of the Pacific proving ground. U.S. Atomic Energy Commission report UWFL-51, 1957.

[7] Welander A. D. Radioactivity in the reef fishes of Belle 1sland, Eniwetok Atoll, April 1954 to November 1955. U.S. Atomic Energy Commission report UWFL-49, 1957.

[8] FontAINE Y. La contamination radioactive des milieux et des organismes aquatiques. Rapport CEA-R-1588, 1960. 
[9] Perkins R. W., Nelson J. L., Haushild W. L. Behavior and transport of radionuclides in the Columbia River between Hanford and Vancouver. Limnol. Oceanogr., 1966, 11, 235-248.

[10] Gustafson P. F. Comments on radionuclides in aquatic ecosystems. In : Radioecological Concentration processes (B. ABERG and F. P. HUnGate, Eds.). Oxford : Pergamon press, 1967, 853-858.

[11] SAIKı M. Études effectuées au Japon dans le domaine de la radioécologie marine. In : Colloque sur la radioécologie marine, Cherbourg, 3-6 décembre 1968. Paris : OCDE, 1968, 83-90.

[12] BAudin J. P. Contribution à l'étude écologique des milieux saumâtres méditerranéens. I : Les principaux caractères physiques et chimiques des eaux de l'étang de Citis (B.-d.-R.). Vie et Milieu, 1981, 30, $\mathrm{n}^{\circ} 2$ (sous presse).

[13] Osterberg C. L., Cutshall N., Johnson V., Cronin J., Jennings D., Frederick L. Some non biological aspects of Columbia River radioactivity. In : Disposal of radioactive wastes into seas, oceans and surface waters, Vienna, 16-20 mai 1966, Vienne; A.I.E.A., 1966, 321-335.

[14] Johnson V., Cutshall N., Osterberg C. L. Retention of ${ }^{65} \mathrm{Zn}$ by Columbia river sediment. Water Resour. Res., 1967, 3, 99-102.

[15] Robertson D. E., Silker W. B., Langford J. C., Petersen M. R., Perkins R. W. Transport and depletion of radionuclides in the Columbia River. In : The radioactive contamination of the marine environment. Seattle, 10-14 July 1972. Vienne : A.I.E.A., 1973, 141-158.

[16] TRIQUET C. Étude de la contamination d'Arenicola marina L. (Annélide Polychète) par le Cobalt 60. C.R. Acad. Sc. Paris, 1973, 276, série D, 645-648.

[17] Amiard-Triquet C. Étude expérimentale de la contamination par le cérium 144 et le fer 59 d'un sédiment à Arénicola marina L. (Annélide Polychète). Cah. Biol. Mar., 1974, 15, 483-493.

[18] Barnes C. A., Gross M. G. Distribution at sea of Columbia river water and its load of radionuclides. In : Disposal of radioactive wastes into seas. oceans and surface waters, Vienna, 12-16 May 1966. Vienne : A.I.E.A., 1966, 291-302.

[19] Aston S. R., Duursma E. K. Concentration effects on ${ }^{137} \mathrm{Cs},{ }^{65} \mathrm{Zn},{ }^{60} \mathrm{Co}$ and ${ }^{106} \mathrm{Ru}$ sorption by marine sediments, with geochemical implications. Neth. J. Sea Res., 1973, 6, 225-240.

[20] Backmann R. W. An experimental study of freshwater zinc cycle. Ph. D. Thesis, Univ. of Michigan, U.S.A., 1961, 79 p.

[21] Duke T. W., Willis J. N., Price T. J. Cycling of trace elements in the estuarine environment. I : Movement and distribution of zinc 65 and stable zinc in experimental ponds. Cheasapeake Sci., $1966,7,1-10$.

[22] Baudin J. P. Premières données sur l'étude expérimentale du cycle du zinc dans l'étang de l'Olivier. Vie Milieu, 1974, 24, 59-80.

[23] Cross F. A., Duke T. W., Will. J. N. Biogeochemistry of trace elements in a coastal plain estuary : distribution of manganese, iron and zinc in sediments, water and Polychaetous worms. Cheasapeake Sci., 1970, 11, 221-234.

[24] VILQUin A., Fowler S. W., ReNFro W. C. Procedures for radioecological studies with marine benthic invertebrates. In : Design of radiotracer experiment in marine biological systems (I.A.E.A. Technical report series $\left.{ }^{\circ} 157\right)$. Vienne : A.I.E.A., 1975, 107-119. 